\title{
IMPERFECT BIFURCATION NEAR A DOUBLE EIGENVALUE: TRANSITIONS BETWEEN NONSYMMETRIC AND SYMMETRIC PATTERNS*
}

\section{T. ERNEUX $\ddagger$ AND D. S. COHEN $\dagger$}

\begin{abstract}
We examine the existence of nonsymmetric and symmetric steady state solutions of a general class of reaction-diffusion equations.

Our study consists of two parts:

(i) By analyzing the bifurcation from a uniform reference state to nonuniform regimes, we demonstrate the existence of a unique symmetric solution (basic wave number two) which becomes linearly stable when it surpasses a critical amplitude. (We assume that the first bifurcation point corresponds to the emergence of the simplest nonsymmetric steady state solutions.)

(ii) This result is not affected when a parameter is nonuniformly distributed in the system. However, one of the two possible branches of nonsymmetric solutions may disappear from the bifurcation diagram.

Our analysis is motivated by the fact that experimental observations of pattern transitions during morphogenesis are interpreted in terms of the dynamics of stable concentration gradients. We have shown that in addition to the values of the physico-chemical parameters, these structures can be selected by two different mechanisms:
\end{abstract}

(i) the linear stability of the nonuniform patterns,

(ii) the effects of a small and nonuniform variation of a parameter in the spatial domain.

1. Introduction. The emergence of spatial patterns from apparently homogeneous layers of identical cells is one of the most difficult problems in developmental biology. It is generally assumed that two distinct processes contribute to their formation: first, the construction of a program which specifies the position of each unit in the cell population (pattern formation); second, the interpretation of this program assigning precise activities to each individual cell (pattern differentiation).

To analyze how a spatial distribution of a physical property may appear inside a population of cells and to find the conditions for its stability, experimental manipulations perturbing the normal development are particularly relevant. For example, in the freshwater coelenterate hydra, small pieces of heads transplanted to the gastric column of hydra induce the formation of a second head there [8], [9]. In eggs of certain insects, the specification of a one-dimensional body pattern can be changed reproducibly in a "double abdomen" pattern by ultraviolet (U.V.) irradiation [14], [24]. From the theoretical point of view, the switch of the normal pattern to different stable structures is particularly complex to describe. Extensive experimental and mathematical studies have demonstrated that these patterns can be associated with stable concentration gradients of chemicals (called morphogens) throughout the morphogenetic fields (see references in [3], [5], [7], [8], [11], [20]-[23], [25]). Thus, we consider the pattern formation as a reaction-diffusion problem. Reaction-diffusion models describe the spatio-temporal evolution of the morphogens as the result of chemical reactions and diffusion processes. They are of the form (for a one-dimensional system of length $l$ ):

$$
\begin{array}{llrl}
\frac{\partial}{\partial t} X & =F(X, \lambda, \mu)+D \frac{\partial^{2} X}{\partial r^{2}}, & & 0 \leqq r \leqq l, \\
\frac{\partial}{\partial r} X & =0, & r & =0, l, \quad X(0, r)=X_{i}(r),
\end{array}
$$

* Received by the editors January 7, 1981, and in revised form October 20, 1982.

$\dagger$ Department of Applied Mathematics, California, Institute of Technology, Pasadena, California 91125.

$\ddagger$ Present address: Department of Engineering Sciences and Applied Mathematics, Northwestern University, Evanston, Illinois 60201. 
where $X=\operatorname{col}\left(X_{1}, X_{2}, \cdots, X_{n}\right)$ is a concentration vector of the chemical intermediates. $F(X, \lambda, \mu)$ represents the reaction kinetics. $D$ is the matrix of constant diffusion coefficients and $\lambda, \mu$ are two control parameters such as rate constants or the concentration of reactants. We assume that $\lambda$ is constant and that $\mu$ admits a spatial dependence of the form:

$$
\mu=\mu(r)=\bar{\mu}+\eta \xi(r), \quad \eta \ll 1,
$$

where $\bar{\mu}$ is a constant. When $\mu=\bar{\mu}$, we also assume that equations (1.1) admit a unique uniform steady state solution:

$$
X=X_{0}(\lambda, \bar{\mu}) .
$$

The paper is presented in two parts. First, we examine the role played by the stability of the nonuniform solutions. We show that there exists a limited number of possible transitions between stable nonsymmetric and symmetric patterns when $\lambda$ is progressively changed $(\eta=0)$. Our analysis is motivated by the fact that several experiments were interpreted in terms of transitions between polar (nonsymmetric) to symmetric chemical gradients [5]. A similar problem is analyzed for nonlinear Marangoni convection [26], [27].

In the second part of the paper, we examine the possibility of bifurcation from (1.3) to nonuniform regimes when $\eta \neq 0$ in (1.2). Physico-chemical parameters are never strictly homogeneous in space, and qualitative modifications of the bifurcating solutions can be expected. Interesting results in this direction have been obtained by Boa and Cohen [2] for a simple model system, and by Fife and Peletier [6] for nonlinear diffusion equations modeling population genetics. We show that the spatial dependence of the parameter $\mu$ can have a more dramatic consequence, as it may supress some steady state patterns and reduce the number of possible transitions.

The paper is organized as follows: in $\S 2$, we analyze the bifurcations from (1.3) to nonuniform steady state solutions of wavenumber 1 and 2 to lowest order when $\mu=\bar{\mu}(\eta=0)$. The method of multiple time expansions is used to calculate the nonuniform states. By contrast to previous studies of double eigenvalues in reactiondiffusion systems [10], [16], we find that the small amplitude long-time regimes may depend on different slow times in the vicinity of certain bifurcation points. Section 3 is devoted to the analysis of the effects of $\mu(r)(\eta \neq 0)$. We demonstrate the existence of a closed branch of nonsymmetric solutions which may disappear as an isola center when the perturbation resulting from the nonuniformity of the parameter $\mu$ surpasses a critical value. Finally, in $\$ 4$ we discuss some further conditions for a correct interpretation of the observed transition between patterns during morphogenesis.

2. Bifurcation to nonsymmeric and symmetric solutions. It will be convenient to define the deviations of concentration from $X=X_{0}$ and the dimensionless space variable $s$ :

$$
x \equiv X-X_{0}, \quad s \equiv r / l .
$$

The reaction-diffusion equations (1.1) with $\mu=\bar{\mu}(\eta=0)$ can be rewritten as:

$$
\begin{aligned}
\frac{\partial}{\partial t} x & =L(\lambda, \bar{\mu}) x+N(x, \lambda, \bar{\mu}), & & 0 \leqq s \leqq 1, \\
\frac{\partial}{\partial s} x & =0, & s & =0,1, \quad x(0, s)=x_{i}(s) .
\end{aligned}
$$


The linear operator $L(\lambda, \bar{\mu})$ is given by:

$$
L(\lambda, \bar{\mu})=\left(\frac{\partial F(X, \lambda, \bar{\mu})}{\partial X_{j}}\right)_{X_{0}}+\left(\frac{D}{l^{2}}\right) \frac{\partial^{2}}{\partial s^{2}}
$$

and $N(x, \lambda, \bar{\mu})$ corresponds to the nonlinear contributions of (1.1) after introducing (2.1).

We assume that when $\lambda$ progressively increases from zero, there exists a domain of $(\lambda, \bar{\mu})$ values where $x=0$ is stable to perturbations uniform in space and the first bifurcation point of the basic state corresponds to nonuniform steady states. A necessary condition for the existence of steady nonuniform solutions of $(2.2)$ is that the following equations admit nontrivial solutions:

$$
L(\lambda, \bar{\mu}) u=0 .
$$

We assume that this is the case when:

$$
\lambda=\lambda_{n}(\bar{\mu}), \quad n=1,2, \cdots .
$$

Furthermore, we assume that the corresponding solutions of (2.4) take the simple form:

$$
u=u_{n}(\bar{\mu})=p_{n} \cos n \pi s, \quad n=1,2, \cdots,
$$

where $p_{n}$ is a constant vector satisfying:

$$
\left\{\left(\partial F(X, \lambda, \bar{\mu}) / \partial X_{j}\right)_{X_{0}, \lambda_{n}}-\left(D / l^{2}\right) n^{2} \pi^{2}\right\} p_{n}=0 .
$$

As is frequently observed in the study of simple two-variable models with nonzero diffusion coefficients [23], we assume that when $n$ is treated as a continuous variable, the functions (2.5) have a unique minimum, and they are unbounded as $n \rightarrow 0$, as shown in Fig. 1. In the context of the linear stability of $x=0$, this simply means that (2.5) represents the neutral stability curve separating values of $\lambda$ where $x=0$ is stable or not with respect to perturbations proportional to $u_{n}$. When the second parameter $\vec{\mu}$ is changed, the relative position of each (primary) bifurcation point $\lambda=\lambda_{n}(\bar{\mu})$ changes. We assume that there exists a critical value $\bar{\mu}=\mu^{0}$ such that:

$$
\lambda_{1}\left(\mu^{0}\right)=\lambda_{2}\left(\mu^{0}\right)=\lambda^{0},
$$

as shown in Fig. 1b. The two corresponding eigenfunctions $u_{k}=u_{k}^{0}(k=1,2)$ defined by (2.6)-(2.7) will then both be in the null space $M$ of $L_{0}=L\left(\lambda^{0}, \mu^{0}\right)$ :

$$
L_{0} u_{k}^{0}=0, \quad k=1,2 .
$$

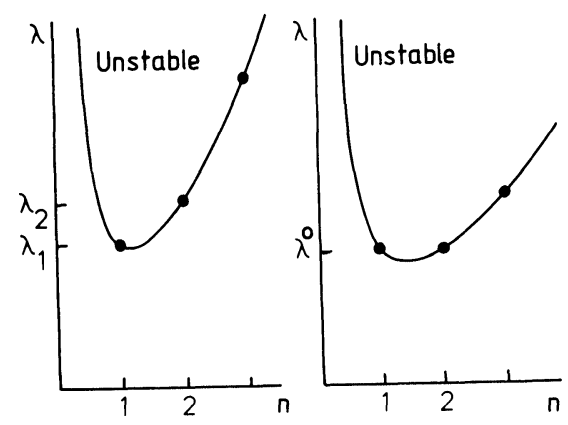

FIG. 1. Linear stability analysis. 
Since we are interested in the stability of the possible nontrivial solutions, we intend to solve the time-dependent problem $(2.2)$ for $(\lambda, \bar{\mu})$ near $\left(\lambda^{0}, \mu^{0}\right)$ using a generalization of the method of Bauer et al., [1], [10], [16], [18]. We define $\gamma \equiv \lambda-\lambda^{0}$ and $\delta \equiv \bar{\mu}-\mu^{0}$ and rewrite equations (2.2) as:

$$
\frac{\partial x}{\partial t}=P(\gamma, \delta) x+Q(x, \gamma, \delta), \quad 0 \leqq s \leqq 1,
$$

$$
\frac{\partial x}{\partial s}=0 \quad s=0,1, \quad x(0, s)=x_{i}(s)
$$

where

(2.10b) $\quad P(\gamma, \delta)=L\left(\lambda^{0}+\gamma, \mu^{0}+\delta\right), \quad Q(x, \gamma, \delta)=N\left(x, \lambda^{0}+\gamma, \mu^{0}+\delta\right)$.

To find an asymptotic expansion of the solutions of (2.10) in the neighborhood of $\gamma=0, \delta=0$ we first define a small parameter $\varepsilon$ by,

$$
\varepsilon=\left|\lambda_{2}(\bar{\mu})-\lambda_{1}(\bar{\mu})\right| \text {. }
$$

It represents the small separation of the two primary bifurcation points when $\gamma$ and $\delta$ deviate from zero. From (2.11) and the definition of $\delta$, it follows that

$$
\delta=\varepsilon \delta_{1}+\varepsilon^{2} \delta_{2}+\cdots
$$

Then we assume the following expansion of the bifurcation parameter $\gamma$ :

$$
\gamma=\varepsilon \gamma_{1}+\varepsilon^{2} \gamma_{2}+\cdots
$$

where $\gamma_{1}, \gamma_{2}, \cdots$ are specified $O(1)$ quantities, and we seek a solution of (2.10) of the form

$$
x=x(t, \tau, s, \varepsilon)=\varepsilon x_{1}(t, \tau, s)+\varepsilon^{2} x_{2}(t, \tau, s)+\cdots
$$

where $\tau$ represents a slow time defined as:

$$
\tau \equiv \varepsilon t
$$

Moreover, we consider small amplitude initial conditions:

$$
x_{i}(s)=\varepsilon x_{1 i}(s)+\varepsilon^{2} x_{2 i}(s)+\cdots .
$$

From (2.14), we find that:

$$
\frac{d}{d t}=\frac{\partial}{\partial t}+\varepsilon \frac{\partial}{\partial \tau}
$$

Furthermore, we assume that $P(\gamma, \delta)$ and $Q(x, \gamma, \delta)$ have the following representations:

$$
P(\gamma, \delta)=L_{0}+\gamma P_{1}+\delta P_{2}+O\left(\gamma^{2}, \gamma \delta, \delta^{2}\right)
$$

when $|\gamma|=O(\delta) \rightarrow 0$,

$$
Q(x, \gamma, \delta)=Q_{2}(x, x, \gamma, \delta)+\cdots+Q_{k}(x, \cdots, x, \gamma, \delta)+\cdots
$$

when $|x| \rightarrow 0 . Q_{k}(x, \cdots, x, \gamma, \delta)(k=2,3, \cdots)$ represent vectors of homogeneous polynomials of $x_{1}, x_{2}, \cdots, x_{n}$ of degree $k$. 
Introducing (2.12), (2.13), (2.15)-(2.18) into (2.10) and equating coefficients of like powers of $\varepsilon$, we obtain the following equations for $x_{1}, x_{2}$ :

$$
\begin{gathered}
\left(\frac{\partial}{\partial t}-L_{0}\right) x_{1}=0, \quad 0 \leqq s \leqq 1, \\
\frac{\partial}{\partial s} x_{1}=0, \quad s=0,1, \quad x_{1}(0,0, s)=x_{1 i}(s) \\
\left(\frac{\partial}{\partial t}-L_{0}\right) x_{2}=\left(\gamma_{1} P_{1}+\delta_{1} P_{2}\right) x_{1}-\frac{\partial}{\partial \tau} x_{1}+Q_{2}\left(x_{1}, x_{1}, 0,0\right), \quad 0 \leqq s \leqq 1, \\
\frac{\partial}{\partial s} x_{2}=0, \quad s=0,1, \quad x_{2}(0,0, s)=x_{2 i}(s) .
\end{gathered}
$$

The general solution of $(2.19)$ is:

$$
x_{1}=\alpha_{1}(\tau) u_{1}^{0}+\alpha_{2}(\tau) u_{2}^{0}+y_{1}(t, \tau, s)
$$

where $u_{k}^{0}(k=1,2)$ are defined by (2.9), $\alpha_{1}(\tau), \alpha_{2}(\tau)$ are two undetermined coefficients and $y_{1}(t, \tau, s)$ is an exponentially decaying function of $t$.

We introduce (2.21) into the right-hand side of (2.20) and apply the solvability conditions. If $u_{k}^{*}=p_{k}^{*} \cos k \pi s(k=1,2)$ denote the two solutions of the adjoint operator to $L_{0}$, these conditions require that [12], [17]

$$
\lim _{T \rightarrow \infty} \frac{1}{T} \int_{0}^{T} d t \int_{0}^{1} d s\left(H(t, \tau, s), u_{k}^{*}(s)\right)=0 \quad(k=1,2)
$$

where $H(t, \tau, s)$ corresponds to the right-hand side of equations (2.20) and $\left(H(t, \tau, s), u_{k}^{*}(s)\right)$ signifies the usual inner product of two vectors. These conditions take the form of two ordinary differential equations:

$$
\begin{array}{ll}
\frac{d \alpha_{1}}{d \tau}=\left[a_{1}\left(\gamma_{1}-q_{1}\right)+A_{2} \alpha_{2}\right] \alpha_{1}, & \frac{d \alpha_{2}}{d \tau}=b_{1}\left(\gamma_{1}-q_{2}\right) \alpha_{2}+B_{2} \alpha_{1}^{2}, \\
\alpha_{1}(0)=\alpha_{1 i}, & \alpha_{2}(0)=\alpha_{2 i},
\end{array}
$$

where $q_{1}, q_{2}$ are defined by,

$$
\begin{aligned}
& q_{1}\left(\delta_{1}\right)=-\delta_{1} a_{2} / a_{1}, \\
& q_{2}\left(\delta_{1}\right)=-\delta_{1} b_{2} / b_{1},
\end{aligned}
$$

and $a_{k}, b_{k}, \alpha_{k i}(k=1,2), A_{2}, B_{2}$ are given by

$$
\begin{aligned}
& a_{1}=\left(P_{1} p_{1}, p_{1}^{*}\right) / N_{1}, \quad a_{2}=\left(P_{2} p_{1}, p_{1}^{*}\right) / N_{1}, \\
& b_{1}=\left(P_{1} p_{2}, p_{2}^{*}\right) / N_{2}, \quad b_{2}=\left(P_{2} p_{2}, p_{2}^{*}\right) / N_{2}, \\
& N_{1}=\left(p_{1}, p_{1}^{*}\right), \quad N_{2}=\left(p_{2}, p_{2}^{*}\right), \\
& A_{2}=\frac{1}{2}\left(Q_{2}\left(p_{1}, p_{2}, 0,0\right)+Q_{2}\left(p_{2}, p_{1}, 0,0\right), p_{1}^{*}\right) / N_{1}, \\
& B_{2}=\frac{1}{2}\left(Q_{2}\left(p_{1}, p_{1}, 0,0\right), p_{2}^{*}\right) / N_{2}, \\
& \alpha_{k i}=\int_{0}^{1} 2 d s\left(x_{1 i}, u_{k}^{*}\right) / N_{k} \quad(k=1,2) .
\end{aligned}
$$

The possible steady states of (2.23) are:

(i)

$$
\bar{\alpha}_{1}=\bar{\alpha}_{2}=0,
$$




$$
\begin{aligned}
& \bar{\alpha}_{1}=0, \quad \gamma_{1}=q_{2}, \quad \bar{\alpha}_{2} \text { undetermined, } \\
& \bar{\alpha}_{2}=-a_{1}\left(\gamma_{1}-q_{1}\right) / A_{2}, \\
& \bar{\alpha}_{1}^{2}=a_{1} b_{1}\left(\gamma_{1}-q_{1}\right)\left(\gamma_{1}-q_{2}\right) / A_{2} B_{2} \geqq 0 .
\end{aligned}
$$

Equation (2.27a) corresponds to the uniform basic state and (2.27b) represents a vertical branch of steady states emerging from the basic state (2.27a) at $\gamma_{1}=q_{2}$. (2.27c) describes solutions which admit two critical points: $\gamma_{1}=q_{1}, \bar{\alpha}_{1}\left(q_{1}\right)=\bar{\alpha}_{2}\left(q_{1}\right)=0$ is a primary bifurcation point and $\gamma_{1}=q_{2}, \bar{\alpha}_{1}\left(q_{2}\right)=0, \bar{\alpha}_{2}\left(q_{2}\right)=-a_{1}\left(q_{2}-q_{1}\right) / A_{2}$ is a secondary bifurcation point: the solution $(2.27 \mathrm{c})$ branches from the solution $(2.27 \mathrm{~b})$ at this point. From (2.23), we can analyze the linear stability of the steady states (2.27):

(i) $(2.27 \mathrm{a})$ is stable when

$$
a_{1}\left(\gamma_{1}-q_{1}\right)<0, \quad b_{1}\left(\gamma_{1}-q_{2}\right)<0 ;
$$

(ii) $(2.27 \mathrm{~b})$ is always unstable when

$$
A_{2} \bar{\alpha}_{2}+a_{1}\left(q_{1}-q_{2}\right)>0
$$

When $A_{2} \bar{\alpha}_{2}+a_{1}\left(q_{1}-q_{2}\right)<0$, the stability properties of $(2.27 \mathrm{~b})$ are not completely determined.

(iii) $(2.27 \mathrm{c})$ is stable when

$$
b_{1}\left(\gamma_{1}-q_{2}\right)<0, \quad A_{2} B_{2}<0 .
$$

The analysis of the next order correction of $(2.27 \mathrm{c})$ shows that it becomes singular when $\gamma_{1} \rightarrow q_{2}$. Therefore we reexamine the bifurcation problem when $\gamma_{1}=q_{2}$. Assuming

$$
\gamma=\varepsilon q_{2}+\varepsilon^{2} \gamma_{2}+\cdots
$$

instead of $(2.12 \mathrm{~b})$, we seek a solution of (2.10) of the form:

$$
\begin{aligned}
x & =x^{*}\left(t, \tau, \tau_{2}, \tau_{3}, s, \varepsilon\right) \\
& =\varepsilon^{1 / 2} x_{1}\left(t, \tau, \tau_{2}, \tau_{3}, s\right)+\varepsilon x_{2}\left(t, \tau, \tau_{2}, \tau_{3}, s\right)+\cdots
\end{aligned}
$$

where $\varepsilon$ and $\tau$ are defined by (2.11) and (2.14), and $\tau_{2}, \tau_{3}$ are two new slow times defined by

$$
\tau_{2}=\varepsilon^{3 / 2} t, \quad \tau_{3}=\varepsilon^{2} t
$$

The expansion (2.30) and the introduction of the new slow times (2.31) are suggested by an analysis of equations (2.23) when $\left|\gamma_{1}-q_{2}\right| \rightarrow 0: \bar{\alpha}_{2}=O(1), \bar{\alpha}_{1}=O\left(\left(\gamma_{1}-q_{2}\right)^{1 / 2}\right)$ and if $\alpha_{j}(\tau)-\bar{\alpha}_{j}(j=1,2)$ remain small, $\alpha_{2}=\alpha_{2}\left(\left(\gamma_{1}-q_{2}\right) \tau\right), \alpha_{1}=\alpha_{1}\left(\left(\gamma_{1}-q_{2}\right)^{1 / 2} \tau\right)$. As previously, we introduce $(2.12 \mathrm{a}),(2.29),(2.30)$ into (2.10) and equate to zero the coefficients of each power of $\varepsilon^{1 / 2}$. By examining the resulting system of equations and their solvability conditions, we find that:

1. To a first approximation, the steady state solutions are given by

$$
x=\varepsilon\left(\beta_{2}+O\left(\varepsilon^{1 / 2}\right)\right) u_{2}^{0}+\varepsilon^{3 / 2} \beta_{1} u_{1}^{0}+O\left(\varepsilon^{2}\right),
$$

where $u_{1}^{0}, u_{2}^{0}$ are defined by (2.9) and $\beta_{2}, \beta_{1}$ satisfy the following conditions:

$$
\left[a_{1}\left(q_{2}-q_{1}\right)+A_{2} \beta_{2}\right] \beta_{1}=0, \quad b_{1}\left(\gamma_{2}-q_{2}^{\prime}\right) \beta_{2}+B_{2} \beta_{1}^{2}+B_{3} \beta_{2}^{3}=0,
$$

where

$$
q_{2}^{\prime}=q_{2}^{\prime}\left(\delta_{2}\right)
$$


and $q_{k}, a_{k}, b_{k}(k=1,2), A_{2}, B_{2}$ are given by (2.24)-(2.26). The new coefficient $B_{3}$ is defined by

$$
\begin{aligned}
B_{3}=\left(3 Q_{3}\left(p_{2}, p_{2}, p_{2}, 0,0\right) / 4\right. & +Q_{2}\left(p_{0}, p_{2}, 0,0\right)+Q_{2}\left(p_{2}, p_{0}, 0,0\right) \\
+ & {\left.\left[Q_{2}\left(p_{4}, p_{2}, 0,0\right)+Q_{2}\left(p_{2}, p_{4}, 0,0\right)\right] / 2, p_{2}^{*}\right) / N_{2}, }
\end{aligned}
$$

where $p_{0}, p_{4}$ are two constant vectors satisfying

$$
L_{0} p_{0}=-Q_{2}\left(p_{2}, p_{2}, 0,0\right) / 2, \quad L_{0} p_{4} \cos 4 \pi s=-Q_{2}\left(p_{2}, p_{2}, 0,0\right) \cos 4 \pi s / 2 .
$$

The solutions of (2.33) are:

$$
\beta_{2}=\beta_{1}=0 \text {, }
$$

$$
\beta_{1}=0, \quad \beta_{2}^{2}=-b_{1}\left(\gamma_{2}-q_{2}^{\prime}\right) / B_{3} \geqq 0,
$$

(iii)

$$
\text { where } \gamma_{2}^{*}=q_{2}^{\prime}-B_{3} \beta_{2}^{* 2} \text {. }
$$

Equation (2.37a) is the basic state; (2.37b) describes a branch of primary steady states emerging at $\gamma_{2}=q_{2}^{\prime}\left(\gamma_{1}=q_{2}\right)$ and corresponds to the perturbation of the vertical branch of solutions $(2.27 \mathrm{~b}) ;(2.37 \mathrm{c})$ describes secondary bifurcating steady states emerging from $(2.37 \mathrm{~b})$ at $\gamma_{2}=\gamma_{2}^{*}\left(\gamma_{1}=q_{2}\right)$ and corresponds to the perturbation of $(2.27 \mathrm{c})$ in the vicinity of the secondary bifurcation point: $\beta_{2}=\beta_{2}^{*}, \beta_{1}=0, \gamma_{2}=\gamma_{2}^{*}$.

2. The linear stability properties of the different steady states (2.37) are:

(i) $(2.37 \mathrm{a})$ is stable when

$$
a_{1}\left(q_{2}-q_{1}\right)<0, \quad b_{1}\left(\gamma_{2}-q_{2}^{\prime}\right)<0 ;
$$

(ii) $(2.37 \mathrm{~b})$ corresponds to stable steady states when

$$
A_{2} \beta_{2}+a_{1}\left(q_{2}-q_{1}\right)<0, \quad B_{3}<0 .
$$

When $\beta_{2}=\beta_{2}^{*}=-a_{1}\left(q_{2}-q_{1}\right) / A_{2},(2.37 \mathrm{~b})$ changes stability and is related to the emergence of $(2.37 \mathrm{c})$.

(iii) $(2.37 \mathrm{c})$ represents stable solutions when

$$
a_{1}\left(q_{2}-q_{1}\right)<0, \quad A_{2} B_{2}<0, \quad b_{1}\left(\gamma_{2}-q_{2}^{\prime}\right)+3 B_{3} \beta_{2}^{* 2}<0 .
$$

If $b_{1}\left(\gamma_{2}-q_{2}^{\prime}\right)+3 B_{3} \beta_{2}^{* 2}=0$, the steady state solutions described by $(2.37 \mathrm{c})$ admit a Hopf bifurcation point to time-periodic solutions.

In summary, we have found that the steady state solutions are, to a first approximation, given by: $x \simeq \bar{\alpha}_{1}^{\prime}(\lambda) u_{1}^{0}+\bar{\alpha}_{2}^{\prime}(\lambda) u_{2}^{0}$ where $\bar{\alpha}_{j}^{\prime}=\varepsilon \bar{\alpha}_{j}(j=1,2)$ when $\lambda-\lambda^{0}=O(\varepsilon)$ or $\bar{\alpha}_{1}^{\prime}=\varepsilon^{3 / 2} \beta_{1}, \bar{\alpha}_{2}^{\prime}=\varepsilon \beta_{2}$ when $\lambda-\lambda^{0}-\varepsilon q_{2}=O\left(\varepsilon^{2}\right)$. Composite bifurcation diagrams can be obtained from the expressions (2.27) and (2.37) for $\bar{\alpha}_{j}, \beta_{j}(j=1,2)$.

In Fig. 2 , we represent the possible diagrams. Figures $2 \mathrm{a}-\mathrm{h}$ present the possible bifurcations from $x=0$ to nontrivial steady state solutions when $\left|\lambda_{2}-\lambda_{1}\right|$ approaches zero. $\bar{\alpha}_{1}^{\prime}+\bar{\alpha}_{2}^{\prime}$ is represented as a function of $\lambda$ for different values of the coefficients $A_{2}, B_{2}, B_{3} . \lambda_{1}, \lambda_{2}$ are primary bifurcation points, whereas $\lambda_{1}^{*}, \lambda_{2}^{*}$ represent secondary bifurcation points $\left(\lambda_{1}^{*}\right.$ corresponds to the bifurcation from $\left(\bar{\alpha}_{1}^{\prime}, \bar{\alpha}_{2}^{\prime}\right) \neq 0$ to time-periodic solutions). Full lines represent stable solutions. Dotted and broken lines correspond to unstable solutions characterized by either two or one eigenvalue in the right half plane. For each case, there exists a symmetric diagram with respect to the $\lambda$ axis which can be obtained by changing the signs of both $A_{2}$ and $B_{2}$. 


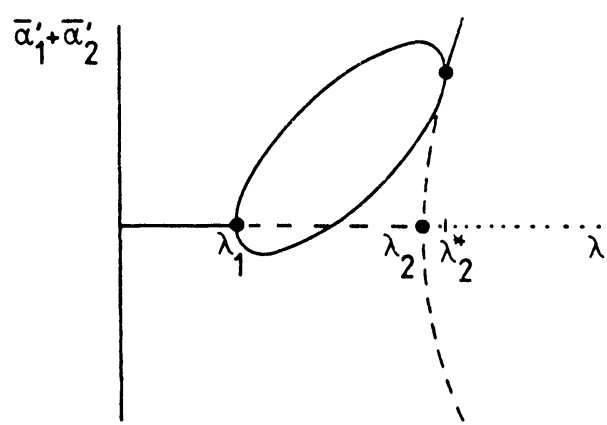

a. $\lambda_{2}>\lambda_{1}, A_{2}<0, B_{2}>0, B_{3}<0$

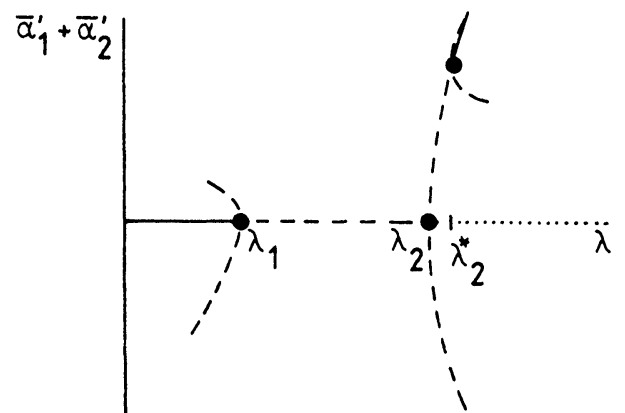

c. $\lambda_{2}>\lambda_{1}, A_{2}<0, B_{2}<0, B_{3}<0$

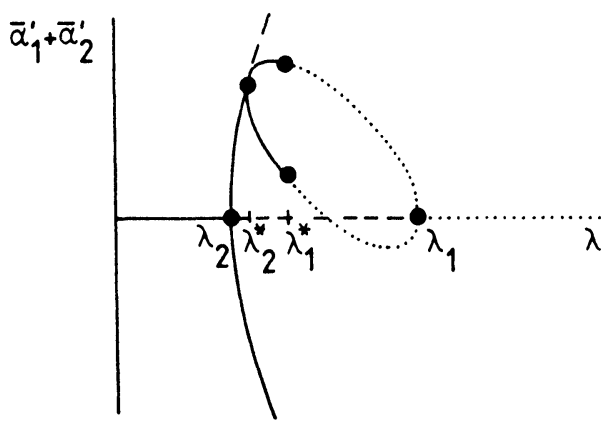

e. $\lambda_{2}<\lambda_{1}, A_{2}>0, B_{2}<0, B_{3}<0$

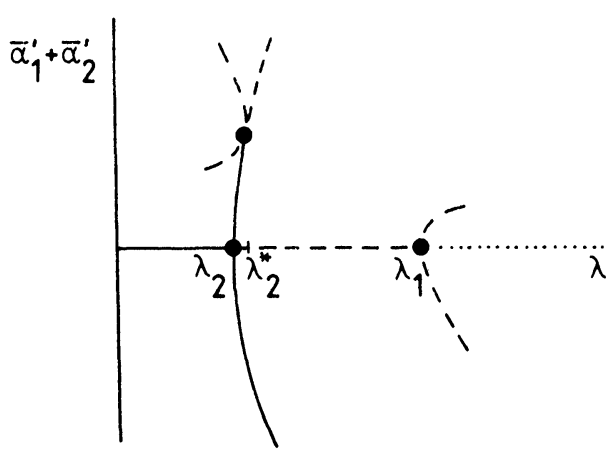

g. $\lambda_{2}<\lambda_{1}, A_{2}>0, B_{2}>0, B_{3}<0$

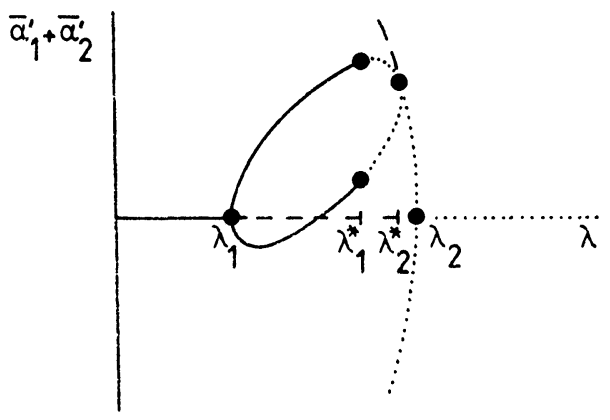

b. $\lambda_{2}>\lambda_{1}, A_{2}<0, B_{2}>0, B_{3}>0$

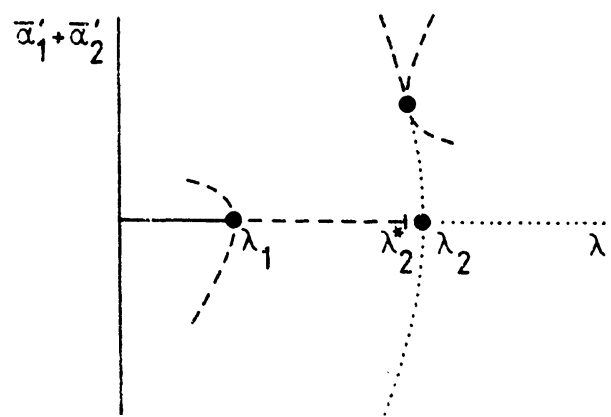

d. $\lambda_{2}>\lambda_{1}, A_{2}<0, B_{2}<0, B_{3}>0$

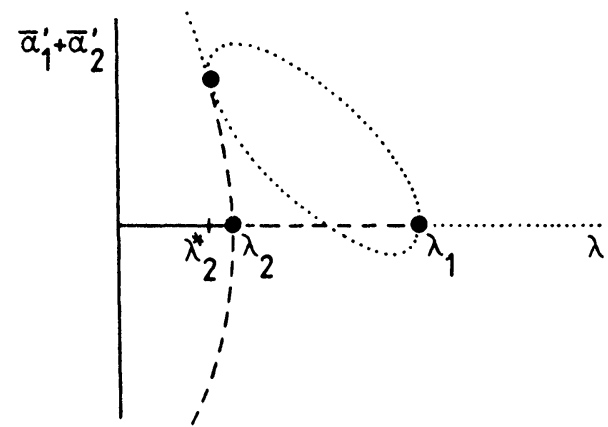

f. $\lambda_{2}<\lambda_{1}, A_{2}>0, B_{2}<0, B_{3}>0$

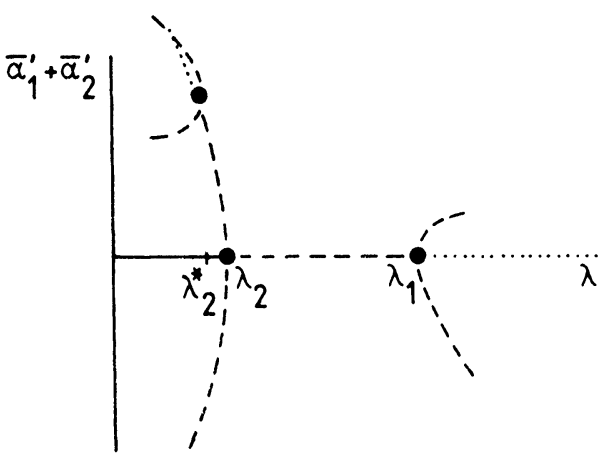

h. $\lambda_{2}<\lambda_{1}, A_{2}>0, B_{2}>0, B_{3}>0$

FIG. 2. Possible bifurcation diagrams of the steady state solutions. 
In Appendix A we show that each case can be observed in a simple chemical model.

3. Imperfect bifurcation to nonsymmetric and symmetric solutions. In this section, we analyze the effect of the spatial variation of $\mu=\mu(s)$ given by (1.2). We assume that $\mu$ appears as a constant term in the kinetic equations (1.1), i.e., that the rate of formation of one of the chemical intermediates follows a zero order kinetics. Taking into account the definitions (1.2), (1.3) and (2.1), the reaction-diffusion equations (1.1) can be rewritten as

$$
\begin{array}{lll}
\frac{\partial x}{\partial t}=L(\lambda, \bar{\mu}) x+\eta \xi(s) L_{1}(\lambda, \bar{\mu})+N(x, \lambda, \bar{\mu}), & 0 \leqq s \leqq 1, & \\
\frac{\partial x}{\partial s}=0, & s=0,1, \quad x(0, s)=x_{i}(s)
\end{array}
$$

where $L(\lambda, \bar{\mu})$ is a matrix defined by $(2.3), L_{1}(\lambda, \bar{\mu})$ is a constant vector and $N(x, \lambda, \bar{\mu})$ is a nonlinear vector of $x$.

We intend to find the steady state solutions of $(3.1)$ for $(\lambda, \bar{\mu})$ near $\left(\lambda^{0}, \mu^{0}\right)$. Thus, we rewrite equations (3.1) with $\partial x / \partial t=0$ in terms of $\gamma$ and $\delta$ :

$$
\begin{array}{ll}
P(\gamma, \delta) x+\eta \xi(s) P_{1}(\gamma, \delta)+Q(x, \gamma, \delta)=0, & 0 \leqq s \leqq 1, \\
\frac{\partial x}{\partial s}=0, & s=0,1,
\end{array}
$$

where $P$ and $Q$ are defined by $(2.10 \mathrm{~b})$ and $P_{1}(\gamma, \delta)=L_{1}\left(\lambda^{0}+\gamma, \mu^{0}+\delta\right)$. Our purpose is to obtain an asymptotic expansion of the solutions of (3.2) in the neighborhood of $\gamma=\delta=\eta=0$. To this end, we assume the following expansion of $\eta$ :

$$
\eta=\varepsilon^{n} c_{n}+\varepsilon^{n+1} c_{n+1}+\cdots
$$

where $\varepsilon$ is defined by (2.11). In general, the solvability condition of the lowest order system in our perturbation analysis requires the condition $n \geqq 2$. In the sequel, we consider the case $n=3$ and $n=2$.

3.1. $\boldsymbol{\eta}=\boldsymbol{O}\left(\varepsilon^{3}\right)$. Our analysis presents three distinct parts: first a general outer expansion, then two inner expansions of the solutions in the vicinity of two critical points.

(i) The outer expansion. We seek a solution of (3.2) of the form

$$
x(s, \varepsilon)=\varepsilon x_{1}(s)+\varepsilon^{2} x_{2}(s)+\cdots .
$$

Introducing (3.3) with $n=3,(3.4)$ and (2.12) into (3.2) and equating to zero the coefficients of each power of $\varepsilon$, we obtain a sequence of linear systems of equations. Their solvability conditions give the following results:

$$
x_{1}(s)=\theta_{1} u_{1}^{0}+\theta_{2} u_{2}^{0},
$$

where $u_{1}^{0}, u_{2}^{0}$ are defined by (2.9). The two amplitudes $\theta_{1}, \theta_{2}$ satisfy the following equations:

$$
\left[a_{1}\left(\gamma_{1}-q_{1}\right)+A_{2} \theta_{2}\right] \theta_{1}=0, \quad b_{1}\left(\gamma_{1}-q_{2}\right) \theta_{2}+B_{2} \theta_{1}^{2}=0,
$$

where $a_{1}, b_{1}, A_{2}, B_{2}$ are defined in (2.26) and $q_{1}, q_{2}$ are given by (2.24), (2.25). Equations (3.6) correspond to the steady form of (2.23), and their solutions admit two critical points $\gamma_{1}=q_{1}, \gamma_{1}=q_{2}$. The analysis of $x_{2}(s)$ shows that the expansion (3.4) becomes singular at these points. Therefore we present inner expansions of the 
solutions of (3.2) in the vicinity of $\gamma_{1}=q_{1}, q_{2}$ and verify that they can be connected to their outer expansion [19].

(ii) Inner expansion for $\gamma_{1}=q_{1}$. The analysis of the outer expansion when $\mid \gamma_{1}-$ $q_{1} \mid \rightarrow 0$ indicates that the singularity occurs when $\gamma_{1}-q_{1}=O\left(\varepsilon^{2 / 3}\right)$ and suggests that the appropriate scalings in this critical regime are

$$
\begin{gathered}
\gamma=\varepsilon q_{1}+\varepsilon^{5 / 3}\left(\Gamma_{1}+\varepsilon^{1 / 3} \Gamma_{2}+\cdots\right), \\
x\left(s, \varepsilon^{1 / 3}\right)=\varepsilon^{4 / 3}\left(X_{1}(s)+\varepsilon^{1 / 3} X_{2}(s)+\cdots\right) .
\end{gathered}
$$

After introducing (3.3) with $n=3,(3.7),(3.8)$ and (2.12a) into (3.2) and equating to zero the coefficients of each power of $\varepsilon^{1 / 3}$, we apply the solvability conditions. We find that

$$
x=\varepsilon^{4 / 3}\left(\phi_{1}+O\left(\varepsilon^{1 / 3}\right)\right) u_{1}^{0}+\varepsilon^{5 / 3} \phi_{2} u_{2}^{0}+O\left(\varepsilon^{2}\right),
$$

where the amplitudes $\phi_{1}, \phi_{2}$ satisfy

$$
K_{1}+a_{1} \Gamma_{1} \phi_{1}+A_{2} \phi_{1} \phi_{2}=0, \quad b_{1}\left(q_{1}-q_{2}\right) \phi_{2}+B_{2} \phi_{1}^{2}=0,
$$

and $K_{1}$ is defined by

$$
K_{1} \equiv 2 \int_{0}^{1} d s\left(c_{3} \xi(s) P_{1}(0,0), u_{1}^{*}(s)\right) / N_{1} .
$$

From (3.10) we find $\Gamma_{1}=\Gamma_{1}\left(\phi_{1}\right)$ and $\phi_{2}=\phi_{2}\left(\phi_{1}\right)$ :

$$
\Gamma_{1}=-\frac{1}{a_{1} \phi_{1}}\left[K_{1}-\frac{A_{2} B_{2} \phi_{1}^{3}}{b_{1}\left(q_{1}-q_{2}\right)}\right], \quad \phi_{2}=-\frac{B_{2} \phi_{1}^{2}}{b_{1}\left(q_{1}-q_{2}\right)} .
$$

Figure 3 represents $\phi_{1}, \phi_{2}$ as functions of $\Gamma_{1}$.

(iii) Inner expansion for $\gamma_{1}=q_{2}$. The asymptotic behavior of the outer expansion when $\left|\gamma_{1}-q_{2}\right| \rightarrow 0$ indicates the nonuniformity of the expansion appears when $\left|\gamma_{1}-q_{2}\right|=$

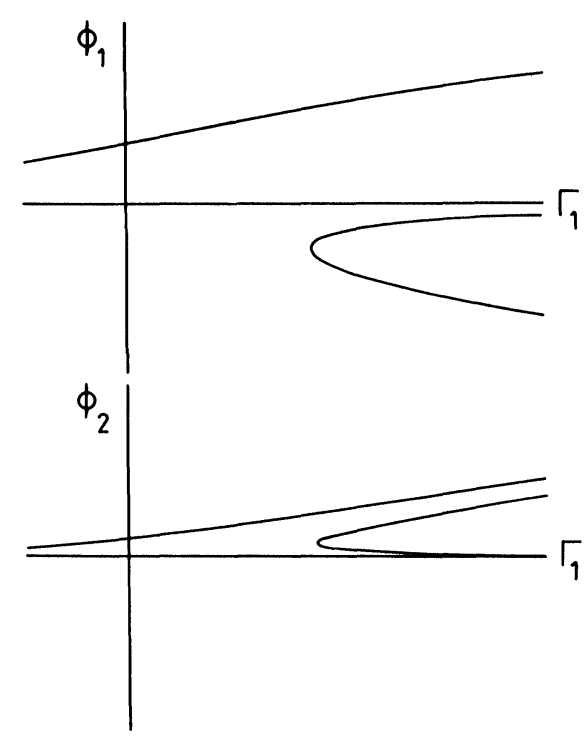

FIG. 3. Inner expansion of the steady state solutions when $\gamma_{1}=q_{1}$. The numerical values of the coefficients appearing in (3.12) used for the picture are: $a_{1}=b_{1}=q_{2}-q_{1}=1, A_{2}=-2, B_{2}=2, K_{1}=1$. 
$O(\varepsilon)$ and suggests that the correct scalings in this regime are:

$$
\begin{gathered}
\gamma=\varepsilon q_{2}+\varepsilon^{2}\left(\Gamma_{1}+\varepsilon \Gamma_{2}+\cdots\right), \\
x\left(s, \varepsilon^{1 / 2}\right)=\varepsilon\left(X_{1}(s)+\varepsilon^{1 / 2} X_{2}(s)+\cdots\right) .
\end{gathered}
$$

We introduce (3.3) with $n=3$, (3.13), (3.14) and (2.12a) into (3.2), equate to zero the coefficients of each power of $\varepsilon^{1 / 2}$ and apply the solvability conditions. We find that:

$$
x=\varepsilon\left(\phi_{2}+O\left(\varepsilon^{1 / 2}\right)\right) u_{2}^{0}+\varepsilon^{3 / 2} \phi_{1} u_{1}^{0}+O\left(\varepsilon^{2}\right),
$$

and the amplitudes $\phi_{1}, \phi_{2}$ satisfy

$$
\left[a_{1}\left(q_{2}-q_{1}\right)+A_{2} \phi_{2}\right] \phi_{1}=0, \quad K_{2}+b_{1}\left(\Gamma_{1}-q_{2}^{\prime}\right) \phi_{2}+B_{2} \phi_{1}^{2}+B_{3} \phi_{2}^{3}=0,
$$

where the new coefficients $q_{2}^{\prime}, B_{3}$ are defined by (2.34), (2.35) and $K_{2}$ is given by

$$
K_{2}=2 \int_{0}^{1} d s\left(c_{3} \xi(s) P_{1}(0,0), u_{2}^{*}(s)\right) / N_{2} .
$$

The possible solutions of equations (3.16) are

$$
\phi_{1}=0, \quad \Gamma_{1}\left(\phi_{2}\right)=q_{2}^{\prime}-\frac{1}{b_{1} \phi_{2}}\left[K_{2}+B_{3} \phi_{2}^{3}\right] ;
$$

(ii) $\quad \phi_{2}=\phi_{2}^{*}=-a_{1}\left(q_{2}-q_{1}\right) / A_{2}, \quad \phi_{1}^{2}\left(\Gamma_{1}\right)=-b_{1} \phi_{2}^{*}\left(\Gamma_{1}-\Gamma_{1}^{*}\right) / B_{2}>0$

$$
\text { where } \Gamma_{1}^{*}=q_{2}^{\prime}-K_{2} / b_{1} \phi_{2}^{*}-B_{3} \phi_{2}^{* 2} / b_{1} \text {. }
$$

Figure 4a represents $\phi_{1}, \phi_{2}$ as functions of $\Gamma_{1}$. The solutions (i) and (ii) admit a common point defined by

$$
\phi_{1}=0, \quad \phi_{2}=\phi_{12}^{*}, \quad \Gamma_{1}=\Gamma_{1}^{*} .
$$
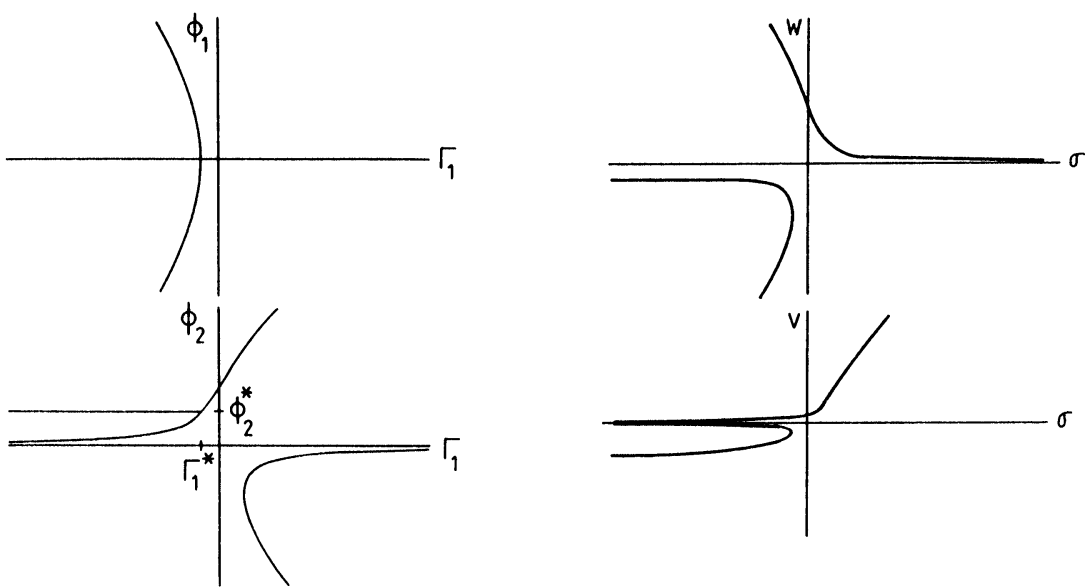

Figs. 4. Inner expansion of the steady state solutions when $\gamma_{1}=q_{2}$. The numerical values of the coefficients appearing in (3.18)-(3.24) used for the picture are: $a_{1}=b_{1}=q_{2}-q_{1}=1, q_{2}^{\prime}=0, A_{2}=-2, B_{2}=2, K_{1}=K_{2}=1$.

However, an analysis of the next order correction of the solutions indicates that they are singular at this point. A new expansion of the solution in the vicinity of (3.20) gives the following results: assuming,

$$
\gamma=\varepsilon q_{2}+\varepsilon^{2} \Gamma_{1}^{*}+\varepsilon^{7 / 3} \sigma+O\left(\varepsilon^{8 / 3}\right),
$$


we find that

$$
x=\varepsilon\left(\phi_{2}^{*}+\varepsilon^{1 / 3} v+O\left(\varepsilon^{2 / 3}\right)\right) u_{2}^{0}+\varepsilon^{5 / 3} w u_{1}^{0}+O\left(\varepsilon^{2}\right),
$$

where $v=v(w)$ and $\sigma=\sigma(w)$ are given by

$$
\begin{gathered}
v(w)=-K_{1} / a_{1} w \\
\sigma(w)=\left[-B_{2} w^{2}+\frac{K_{1}}{a_{1} w}\left(\Gamma_{1}^{*}-q_{2}^{\prime}+3 B_{3} \phi_{2}^{* 2}\right)\right] / b_{1} \phi_{2}^{*} .
\end{gathered}
$$

Figure $4 \mathrm{~b}$ represents $w$ and $v$ as functions of $\sigma$.

Taking into account the behavior of the solutions near (3.20), a composite picture of the inner solution when $\gamma_{1}=q_{2}$ can be drawn. On the other hand, a uniform representation of the steady state solutions can be formed from the inner and outer expansion [19] but will not be presented here.

3.2. $\boldsymbol{\eta}=\boldsymbol{O}\left(\boldsymbol{\varepsilon}^{2}\right)$.

(i) The outer expansion. In this case, we seek a solution of (3.2) of the form (3.4). Introducing (3.3) with $n=2,(3.4)$ and (2.12) into (3.2) and equating to zero the coefficients of each power of $\varepsilon$, we find from the solvability conditions that $x_{1}(s)$ is given by (3.5) where the amplitudes $\theta_{1}, \theta_{2}$ satisfy

$$
\begin{gathered}
K_{1}+a_{1}\left(\gamma_{1}-q_{1}\right) \theta_{1}+A_{2} \theta_{1} \theta_{2}=0, \\
K_{2}+b_{1}\left(\gamma_{1}-q_{2}\right) \theta_{2}+B_{2} \theta_{1}^{2}=0
\end{gathered}
$$

where $K_{1}, K_{2}$ are defined by (3.11), (3.17) with $c_{2}$ instead of $c_{3}$. When $\gamma_{1} \neq q_{2}, \theta_{1}\left(\gamma_{1}\right)$ verifies the following cubic equation:

$$
F\left(\theta_{1}, \gamma_{1}\right)=-A_{2} B_{2} \theta_{1}^{3}+\theta_{1}\left[a_{1} b_{1}\left(\gamma_{1}-q_{1}\right)\left(\gamma_{1}-q_{2}\right)-A_{2} K_{2}\right]+K_{1} b_{1}\left(\gamma_{1}-q_{2}\right)=0
$$

and knowing $\theta_{1}, \theta_{2}\left(\gamma_{1}\right)$ can be obtained from (3.25b): $\theta_{2}=-\left(K_{2}+B_{2} \theta_{1}^{2}\right) / b_{1}\left(\gamma_{1}-q_{2}\right)$. On the other hand when $\sigma=\gamma_{1}-q_{2} \rightarrow 0$, we find that the solutions of (3.25) are:

$$
\begin{gathered}
\theta_{1}=\frac{K_{1} b_{1} \sigma}{K_{2} A_{2}}+O\left(\sigma^{2}\right), \quad \theta_{2}=-\frac{K_{2}}{\sigma}+I(1) \\
\text { (ii) if } K_{2} / B_{2}<0, \quad \theta_{1}^{2}=-\frac{K_{2}}{B_{2}}+O(\sigma), \quad \theta_{2}=-\frac{\left[K_{1}+a_{1}\left(q_{2}-q_{1}\right) \theta_{1}\right]}{A_{2} \theta_{1}}+O(\sigma) .
\end{gathered}
$$

(ii) Inner expansion for $\gamma_{1}=q_{2}$. Since $\theta_{2}$ becomes singular in (3.27), we need an inner expansion of the solutions in the vicinity of $\gamma_{1}=q_{2}$. We find that the appropriate scalings in this critical region are:

$$
\begin{gathered}
\gamma=\varepsilon q_{2}+\varepsilon^{4 / 3}\left(\Gamma_{1}+\varepsilon^{1 / 3} \Gamma_{2}+\cdots\right), \\
x\left(s, \varepsilon^{1 / 3}\right)=\varepsilon^{2 / 3}\left(X_{1}(s)+\varepsilon^{1 / 3} X_{2}(s)+\varepsilon^{2 / 3} X_{3}(s)+\cdots\right),
\end{gathered}
$$

and we obtain the following results:

$$
x=\varepsilon^{2 / 3}\left(\phi_{2}+O\left(\varepsilon^{1 / 3}\right)\right) u_{2}^{0}+\varepsilon^{4 / 3}\left[\phi_{1} u_{1}^{0}+\phi_{2}^{2}\left(p_{0}+p_{4} \cos 4 \pi s\right)\right]+O\left(\varepsilon^{5 / 3}\right),
$$

where $p_{0}, p_{4}$ are defined by (2.36) and $\phi_{1}, \phi_{2}$ satisfy:

$$
K_{1}+A_{2} \phi_{1} \phi_{2}=0, \quad K_{2}+b_{1} \Gamma_{1} \phi_{2}+B_{3} \phi_{2}^{3}=0
$$

The solution of equations (3.32) can be described by $\Gamma_{1}\left(\phi_{2}\right), \phi_{1}\left(\phi_{2}\right)$ :

$$
\Gamma_{1}\left(\phi_{2}\right)=-\frac{1}{b_{1} \phi_{2}}\left[K_{2}+B_{3} \phi_{2}^{3}\right], \quad \phi_{1}\left(\phi_{2}\right)=-\frac{K_{1}}{A_{2} \phi_{2}} \text {. }
$$


Composite expansion of the solutions can be constructed from the inner and outer expansions, but will not be presented here.

From the outer description given by the roots of (3.26), we note the possible existence of a closed branch of solutions (isola). See Fig. 5. The conditions for an isola center have been studied recently [4]. They can be obtained by solving the following three equations:

$$
\begin{aligned}
& F\left(\theta_{1}, \gamma_{1}\right)=0, \\
& \frac{\partial F}{\partial \theta_{1}}\left(\theta_{1}, \gamma_{1}\right)=0, \\
& \frac{\partial F}{\partial \gamma_{1}}\left(\theta_{1}, \gamma_{1}\right)=0 .
\end{aligned}
$$

These equations can easily be solved numerically. Figure 6 gives the locus of the isola centers in $\left(K_{1}, K_{2}\right)$ space for fixed values of the other parameters. The inner region of the triangle corresponds to parameter values associated with isolas.

In conclusion, we have shown that the imperfection resulting from a small nonuniform variation of a parameter can modify considerably the bifurcation diagram of the steady state solutions near a double eigenvalue. Defining $\varepsilon$ as the separation of the two primary bifurcation points and $\eta$ as the order of magnitude of the imperfection, we found that when $\eta=O\left(\varepsilon^{2}\right)$ the bifurcating solutions are only perturbed in the vicinity of the primary bifurcation points. However, when $\eta=O\left(\varepsilon^{2}\right)$, branches of nonsymmetric steady state solutions may disappear as isolas.

4. Discussion. A large number of theoretical studies have been devoted to the analysis of the morphogenetic processes as simple transitions between concentration
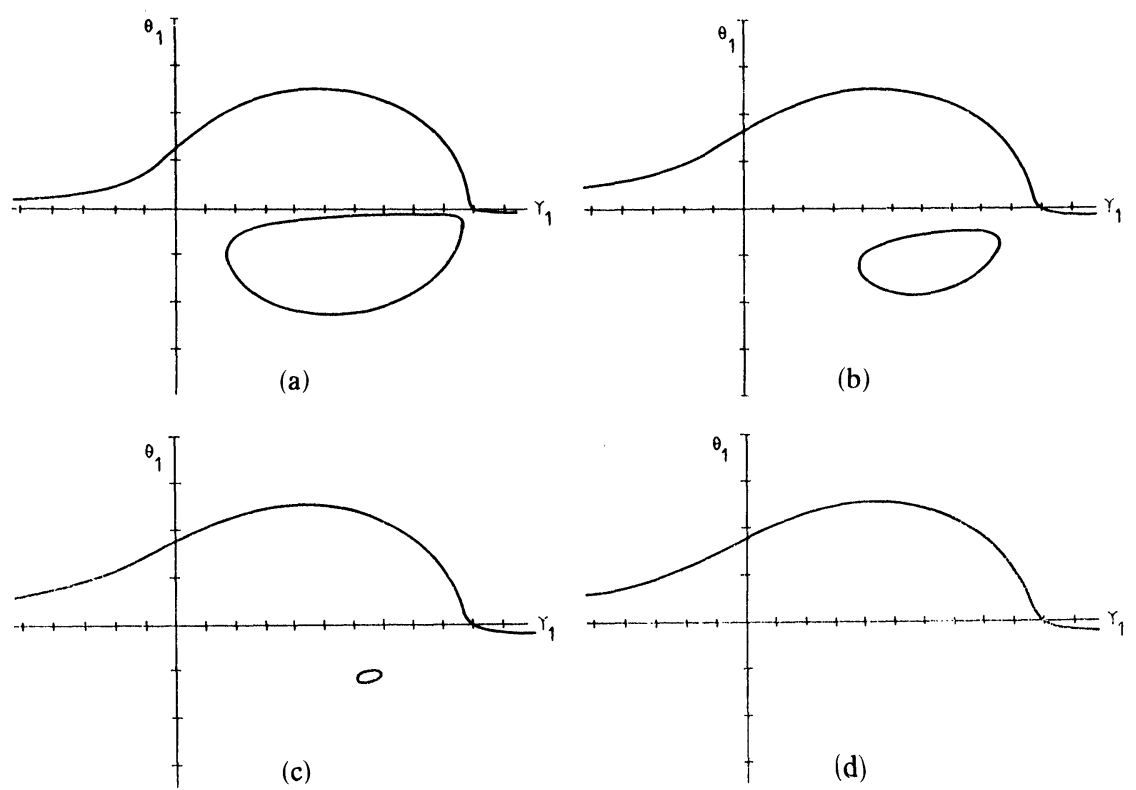

FIGS. 5. The isolas. $\theta_{1}$ is represented as a function of $\gamma_{1}$ and is obtained by solving equation (3.26). The values of the coefficients appearing in this equation are: $a_{1}=b_{1}=q_{2}=1, q_{1}=0, A_{2}=-2, B_{2}=2$ and $K_{1}=K_{2}=10^{-2}$ (Fig. 5a); $K_{1}=K_{2}=2.5310^{-2}$ (Fig. 5b); $K_{1}=K_{2}=3.4310^{-2}$ (Fig. 5c); $K_{1}=K_{2}=3.5210^{-2}$ (Fig. 5d). The unities are equal to 0.1 for both axes. 


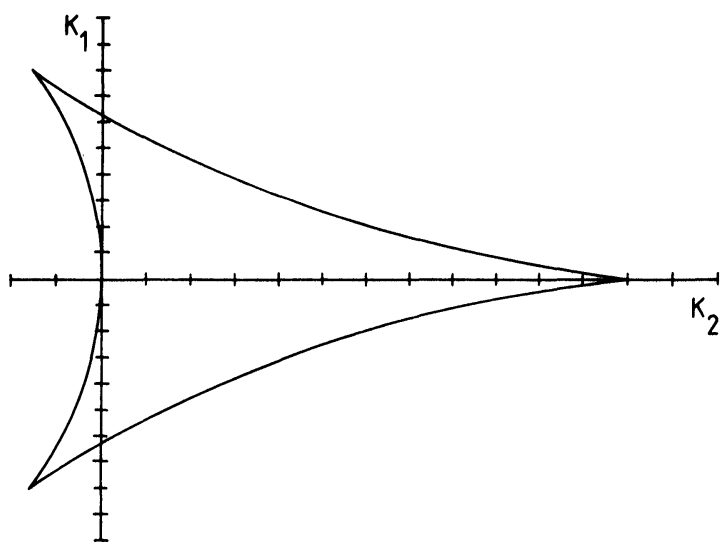

FIG. 6. Locus of the isola points in $\left(K_{1}, K_{2}\right)$ parameter space. $A_{2}=-2, B_{2}=2$. The inner region of the triangle corresponds to isola solutions. The unities are equal to $10^{-2}$ for both axes.

gradients. These structures result, to first approximations, from the combined effects of nonlinear chemical reactions and transport processes and depend on one or several control parameters. In many experiments, a deliberate perturbation modifies specifically the normal developmental program by changing these key parameters in an irreversible way. For example, in the case of insect embryogenesis, the polar pattern (cephalon-abdomen) switches either to an inverse polarity or to a "mirror-symmetric" structure (abdomen-abdomen) [14]. The strategy of a succession of well-defined gradients corresponding to precise values of a control parameter has also been proposed by Kauffman et al. [15] for the development of the imaginal discs of Drosophila. Here, it is the size of the spatial system which acts as the bifurcation parameter.

Our main interest was concentrated on the existence of steady state patterns, solution of the reaction-diffusion equations which present 1 or 2 as characteristic wave numbers. We explore the bifurcation possibilities of a uniform reference state when a parameter $\lambda$ progressively increases from zero and in the vicinity of a double eigenvalue, defined by the critical values of two parameters: $\lambda=\lambda^{0}, \bar{\mu}=\mu^{0}$.

When the uniform reference state presents a first bifurcation to nonsymmetric solutions $\left(q_{2}>q_{1}\right)$, we have found:

(i) It is possible to observe two stable solutions characterized by a basic wave number 1 (polar structures) but only one stable solution of basic wave number 2 (symmetric or duplicate structure) (Fig. 2a). The transition between the two types of solutions is possible by a simple variation of the bifurcation parameter.

(ii) Other behaviors may be expected when the bifurcation parameter gradually changes: jumps to possible $O(1)$ states not described by our analysis (Figs. 2c, 2d) or transition from a nonsymmetric steady state to a time-periodic solution of large period (Fig. 2b).

When the uniform reference steady state presents a first bifurcation to symmetric solutions $\left(q_{2}<q_{1}\right)$, different conclusions appear:

(i) In the vicinity of the first primary bifurcation point, two stable symmetric solutions can be observed. However, one of the two branches of symmetric solutions becomes unstable when the bifurcation parameter increases (Fig. 2f); and

(ii) By contrast to the previous situation (Fig. 2b), the Hopf bifurcation point is a tertiary bifurcation point (Fig. 2e). 
In the presence of a nonuniform variation of a physico-chemical parameter (called pre-pattern, see [8]), the bifurcation diagram of the possible steady states presents considerable changes: if the imperfection surpasses a critical threshold, a complete branch of nonsymmetric steady states can disappear. however, it is still possible to observe a transition from a nonsymmetric solution to a quasisymmetric solution when the bifurcation parameter gradually varies. The sequence of Figs. 7a-e illustrates the gradual transformation of the bifurcation diagram when the size of the imperfection increases. Assuming that $x=\sum_{j=1}^{\infty} \bar{\alpha}_{j}^{\prime} p_{j} \cos j \pi s$, we represent the amplitude of the two principal modes as a function of $\lambda$. The same units on the $\bar{\alpha}_{1}^{\prime}, \bar{\alpha}_{2}^{\prime}$ axis indicate the relative scale of the two amplitudes.
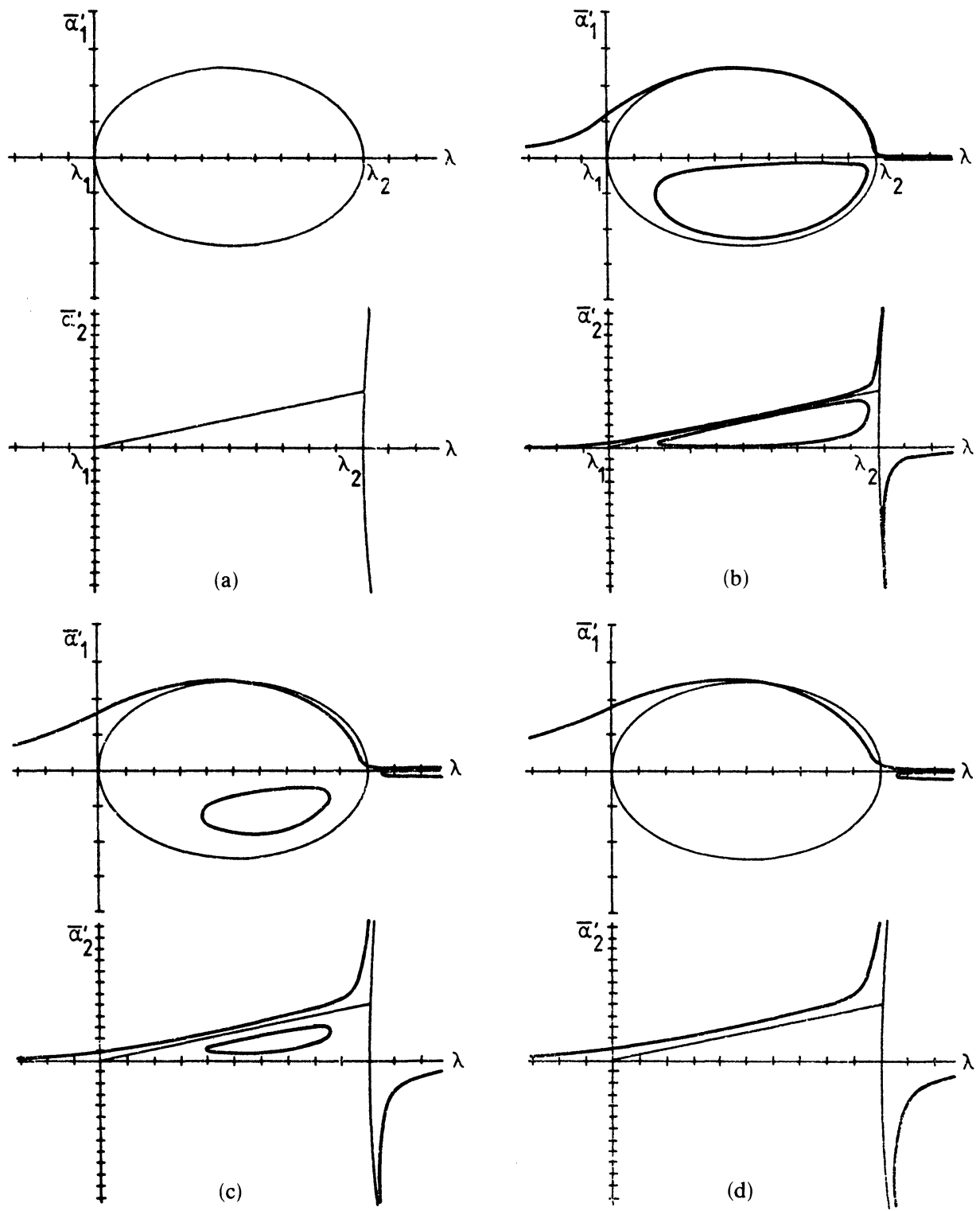

FIGs. 7. Imperfect bifurcation. The bifurcation diagrams for the amplitudes $\bar{\alpha}_{1}^{\prime}, \bar{\alpha}_{2}^{\prime}$ have been drawn showing the gradual change of the distinct branches of solutions when the size of the imperfection increases. Fig. 7a gives the bifurcating solutions. 
As a consequence, our results predict that the inversion of polarity experiment (transition between the two distinct branches of nonsymmetric solutions at a fixed value of the bifurcation parameter and resulting from an initial perturbation of the chemical intermediates) is not always possible whereas the duplication experiment (transition from a nonsymmetric to a symmetric solution when the bifurcation parameter progressively changes) can be successful. A discussion of the experimental data in agreement with our theoretical analysis is given in [5] and [13].

Appendix A. The Brussellator model. The Brussellator is a two-variable chemical model described by the following evolution equations:

(A.1)

$$
\begin{aligned}
& \frac{\partial X}{\partial t}=A-(B+1) X+X^{2} Y+D_{1} \frac{\partial^{2} X}{\partial^{2} s} \\
& \frac{\partial Y}{\partial t}=B X-X^{2} Y+D_{2} \frac{\partial^{2} Y}{\partial^{2} s}, \quad 0<s<1, \\
& \frac{\partial X}{\partial s}=\frac{\partial Y}{\partial s}=0, \quad s=0,1, \\
& X=X_{i}(s), \quad Y=Y_{i}(s), \quad t=0 .
\end{aligned}
$$

$X, Y$ represent the chemical intermediates of the global reaction $A+B \rightarrow P$ [23]. $A$, $B$ are two input products and $D_{j} \equiv D_{j}^{\prime} / l^{2}(j=1,2)$ where $D_{1}^{\prime}, D_{2}^{\prime}$ are two constant diffusion coefficients and $l$ is the length of the system. We assume that $B$ is our bifurcation parameter and $A=A(s)$.

A.1. $\boldsymbol{A}=\overline{\boldsymbol{A}}=$ cst. Equations (A.1) admit a unique steady state solution which is uniform in space:

$$
X_{0}=\bar{A}, \quad Y_{0}=B / \bar{A} .
$$

When

$$
B=\bar{B}_{n}(\bar{A})=1+\bar{A}^{2} D_{1} / D_{2}+\bar{A}^{2} / D_{2} n^{2} \pi^{2}+D_{1} n^{2} \pi^{2}, \quad n=1,2, \cdots,
$$

and

$$
\bar{B}_{n}(\bar{A})<1+\bar{A}^{2}, \quad n=1,2, \cdots,
$$

the system presents a bifurcation from (A.2) to a nonuniform steady state with a basic wave number equal to $n$. Moreover, if,

$$
\bar{A}=A^{0}=\left(D_{1} D_{2} 4 \pi^{4}\right)^{1 / 2}, \quad B=B^{0}=1+D_{1} 5 \pi^{2}+4 D_{1}^{2} \pi^{4}
$$

and

$$
5+4 D_{1} \pi^{2}<D_{2} 4 \pi^{2}
$$

the first bifurcation point corresponds to a double eigenvalue, i.e.,

$$
B^{0}=\bar{B}_{1}\left(A^{0}\right)=\bar{B}_{2}\left(A^{0}\right) \text {. }
$$

We now examine the "splitting" of the double eigenvalue; by defining

$$
\varepsilon=\left|\bar{B}_{2}(\bar{A})-\bar{B}_{1}(\bar{A})\right|<1
$$

as the separation between the primary bifurcation points $B_{1}, B_{2}$, it follows that

$$
\bar{A}=A^{0}+\varepsilon \delta_{1}+\varepsilon^{2} \delta_{2}+\cdots
$$


where

$$
\delta_{1}=-e A^{0} / 6 D_{1} \pi^{2}, \quad \delta_{2}=-A^{0} / 72 D_{1}^{2} \pi^{4}
$$

and $e= \pm 1$ when $B_{2}-B_{1} \gtrless 0$. Moreover, we assume the following expansion of $B$ :

$$
B=B^{0}+\varepsilon \gamma_{1}+\varepsilon^{2} \gamma_{2}+\cdots
$$

where $\gamma_{1}, \gamma_{2}$ are specified $O(1)$ quantities. Then we seek a solution of (A.1) of the form:

$$
\begin{aligned}
& X(t, \tau, \varepsilon, s)=\bar{A}(\varepsilon)+\varepsilon x_{1}(\tau, t, s)+\varepsilon^{2} x_{2}(\tau, t, s)+\cdots, \\
& Y(t, \tau, \varepsilon, s)=B(\varepsilon) / \bar{A}(\varepsilon)+\varepsilon y_{1}(\tau, t, s)+\varepsilon^{2} y_{2}(\tau, t, s)+\cdots,
\end{aligned}
$$

where

$$
\tau \equiv \varepsilon t .
$$

After introducing the expansions (A.9)-(A.12) into (A.1) and equating to zero the coefficients of each power of $\varepsilon$, we examine the solvability conditions. In first approximation, we find that:

$$
\left(\begin{array}{l}
x_{1} \\
y_{1}
\end{array}\right) \simeq \alpha_{1}(\tau)\left(\begin{array}{c}
1 \\
C_{1}
\end{array}\right) \cos \pi s+\alpha_{2}(\tau)\left(\begin{array}{c}
1 \\
C_{2}
\end{array}\right) \cos 2 \pi s, \quad t \rightarrow \infty
$$

where

$$
C_{1}=-\left(1+D_{1} \pi^{2}\right) / D_{2} \pi^{2}, \quad C_{2}=-\left(1+D_{1} 4 \pi^{2}\right) / D_{2} 4 \pi^{2}
$$

and $\alpha_{1}, \alpha_{2}$ satisfy the amplitude equations (2.23) where,

$$
\begin{aligned}
& a_{1}=\left\{\left[D_{2}-4 D_{1}+D_{1} 4 \pi^{2}\left(D_{2}-D_{1}\right)\right] / D_{2}\right\}^{-1}, \\
& b_{1}=\left\{\left[4 D_{2}-D_{1}+4 D_{1} \pi^{2}\left(D_{2}-D_{1}\right)\right] / D_{2}\right\}^{-1},
\end{aligned}
$$

$$
q_{j}=-\frac{e}{3}\left(D_{1} 4 \pi^{2}+4 / j^{2}\right), \quad j=1 \text { or } 2,
$$

$$
\begin{gathered}
A_{2}=a_{1}\left(1-4 D_{1}^{2} \pi^{4}\right) / A^{0}, \\
B_{2}=b_{1}\left(D_{1} \pi^{2}+1\right)\left(1-4 D_{1} \pi^{2}\right) / 2 A^{0} .
\end{gathered}
$$

Using the condition (A.6), it can be shown that

$$
a_{1}>0, \quad b_{1}>0 \text {. }
$$

On the other hand, since

$$
\bar{B}_{j}(\varepsilon)=B^{0}+\varepsilon e q_{j}, \quad j=1 \text { or } 2,
$$

Expression (A.17) corresponds to the primary bifurcation points. The possible bifurcation diagrams depend on the sign of $A_{2}, B_{2}$. From (A.18)-(A.20), we find the results shown in Table 1 . As explained in $\S 2$, an additional analysis is required when $\gamma_{1}=q_{2}$. The description of the bifurcation diagram in that case needs the knowledge of $q_{2}^{\prime}$

TABLE 1 .

\begin{tabular}{rcc}
\hline$D_{1}$ & $A_{2}$ & $B_{2}$ \\
\hline$D_{1}<\frac{1}{4} \pi^{2}$ & + & + \\
$\frac{1}{4} \pi^{2}<D_{1}<\frac{1}{2} \pi^{2}$ & + & - \\
$D_{1}>\frac{1}{2} \pi^{2}$ & - & - \\
\hline
\end{tabular}


and the sign of $B_{3}$ defined by (2.34) and (2.35). We find

$$
q_{2}^{\prime}=0
$$

and

$$
B_{3} \gtrless 0 \quad \text { if } 32 D_{1}^{2} \pi^{4}-81 D_{1} \pi^{2}+4 \gtrless 0 .
$$

TABLE 2.

\begin{tabular}{cc}
\hline$D_{1}$ & $B_{3}$ \\
\hline$D_{1}<0.05 / \pi^{2}$ & + \\
$0.05 / \pi^{2}<D_{1}<2.48 / \pi^{2}$ & - \\
$D_{1}>2.48 / \pi^{2}$ & + \\
\hline
\end{tabular}

Consequently, we have the possibilities displayed in Table 3 , where the $*$ denotes the symmetric diagram with respect to the $\lambda$ axis.

\begin{tabular}{|c|c|c|c|c|c|c|c|c|c|}
\hline$D_{1} \pi^{2}$ & 0.05 & & 0.25 & & 0.5 & & 2.48 & & \\
\hline$B_{3}$ & 0 & - & & - & & - & 0 & + & \\
\hline$A_{2}$ & & + & & + & 0 & - & & - & \\
\hline$B_{2}+$ & & + & 0 & - & & - & & - & \\
\hline Fig. $2 \mathrm{~d}^{*}$ & & $2 c^{*}$ & & $2 a^{*}$ & & $2 c$ & & $2 d$ & $(e>0)$ \\
\hline Fig. $2 \mathrm{~h}$ & & $2 \mathrm{~g}$ & & $2 \mathrm{e}$ & & $2 \mathrm{~g}^{*}$ & & $2 h^{*}$ & $(e<0)$ \\
\hline
\end{tabular}

TABLE 3.

A.2. $\boldsymbol{A}=\boldsymbol{A}(\boldsymbol{s})$. We now examine the case of a small nonuniform variation of $A(s)$. Assuming that

$$
A(s)=\bar{A}+\eta a(s),
$$

the bifurcation diagram of the possible steady states is described in $\S 3$ where the new coefficients $K_{1}, K_{2}$ are given by

$$
\left\{\begin{array}{l}
K_{1} \\
K_{2}
\end{array}=4 A^{0} c_{k}\left\{\begin{array} { l } 
{ a _ { 1 } } \\
{ b _ { 1 } }
\end{array} \cdot \int _ { 0 } ^ { 1 } d s a ( s ) \left\{\begin{array}{l}
\cos \pi s \\
\cos 2 \pi s
\end{array}\right.\right.\right.
$$

where $c_{k}(k=3$ or 2$)$ is related to the expansion of $\eta$ in powers of $\varepsilon$, given by (3.3).

Acknowledgments. We thank Professor E. L. Reiss for many suggestions and for the critical reading of the manuscript. T.E. is "Chargé de Recherches" of F.N.R.S. (Belgium).

\section{REFERENCES}

[1] L. BAuer, H. B. Keller AND E. L. Reiss, Multiple eigenvalues lead to secondary bifurcation, SIAM Rev., 17 (1975), pp. 101-122.

[2] J. A. BOA AND D. S. COHEN, Bifurcation of localized disturbances in a model biochemical reaction, this Journal, 30 (1976), pp. 123-135.

[3] B. Bunow, J. P. Kernevez, G. Joly and D. Thomas, Pattern formation by reaction-diffusion instabilities: Application to morphogenesis in Drosophila, J. Theoret. Biol., 84 (1980), pp. 629-650.

[4] D. Dellwo, H. B. Keller, B. J. Matkowsky AND E. L. Reiss, On the birth of isolas, this Journal, 42 (1982), pp. 956-963.

[5] T. ERneuX And J. HiernauX, Transition from polar to duplicate patterns, J. Math. Biol., 9 (1980), pp. 193-211.

[6] P. C. Fife AND L. Peletier, Nonlinear diffusion in population genetics, Arch. Rational Mech. Anal., 64 (1977), pp. 93-109. 
[7] P. C. FIFE, Mathematical Aspects of Reacting and Diffusing Systems, Lecture Notes in Biomathematics, 28, Springer-Verlag, Berlin, 1979.

[8] A. GIERER, Biological features and physical concepts of pattern formation exemplified by Hydra, Curr. Top. Develop. Biol., 11 (1977), pp. 17-59.

[9] - Generation of biological patterns and form: Some physical, mathematical and logical aspects, Progr. Biophys. Molecular Biol., 37 (1981), pp. 1-47.

[10] R. A. Goldstein, M. A. Huerta AND J. C. NeARing, Secondary bifurcation near multiple eigenvalues, 1983, to appear.

[11] B. C. Goodwin, Analytical Physiology of Cells and Developing Organisms, Academic Press, New York, 1976.

[12] G. J. HABETLER AND B. J. MATKowsky, On the validity of a nonlinear dynamic stability theory, Arch. Rational Mech. Anal., 57 (1975), pp. 166-188.

[13] J. Hiernaux ANd T. ERneux, Pattern selection during morphogenesis, in Proc. of Conf. on Instabilities, Bifurcations and Fluctuations in Chemical Systems, Univ. of Texas Press, Austin, TX, 1982, pp. 199-225.

[14] K. KAlthoff, Analysis of a morphogenetic determinant in an insect embryo, Symp. Soc. Develop. Biol., 37 (1979), pp. 97-126.

[15] S. A. Kauffman, R. M. Shymko And K. Trabert, Control of sequential compartment formation in Drosophila, Science, 199 (1978), pp. 259-270.

[16] J. P. KEENER, Secondary bifurcation in nonlinear diffusion reaction equations, Stud. Appl. Math., 55 (1976), pp. 187-211.

[17] S. KOGELMAN AND J. B. KELLER, Transient behavior of unstable nonlinear systems with applications to the Benard and Taylor problems, this Journal, 20 (1971), pp. 619-637.

[18] T. J. MAhAR AND B. J. MATKowsky, A model biochemical reaction exhibiting secondary bifurcation, this Journal, 32 (1977), pp. 394-404.

[19] B. J. MATKOWSKY AND E. L. REISs, Singular perturbations of bifurcations, this Journal, 33 (1977), pp. 230-255.

[20] H. MEINHARDT, Models for the ontogenetic development of higher organisms, Rev. Physiol. Biochem. Pharmacol., 80 (1978), pp. 48-104.

[21] J. D. Murray, A prepattern formation mechanism for animal coat markings, J. Theoret. Biol., 88 (1981), pp. 161-199.

[22] S. A. NEWMAN AND H. L. FRISCH, Dynamics of skeletal pattern formation in developing chick limb, Science, 205 (1979), pp. 662-668.

[23] G. NiCOlis AND I. Prigogine, Self-Organization in Non-Equilibrium Systems, John Wiley, New York, 1977.

[24] K. SANDER, Specification of the basic body pattern in insect embryogenesis, Adv. Insect Physiol., 12 (1976), pp. 125-238.

[25] L. Wolpert, Pattern formation in biological development, Scientific American, October 1978, pp. 124-137.

[26] S. Rosenblat, S. H. Davis AND G. M. Homsy, Nonlinear Marangoni convection in bounded layers. Part 1. Circular cylindrical containers, J. Fluid Mech., 120 (1982), pp. 91-122.

[27] S. Rosenblat, G. M. Homsy AND S. H. DAvis, Nonlinear Marangoni convection in bounded layers. Part 2. Rectangular cylindrical containers, J. Fluid Mech., 120 (1982), pp. 123-138. 